\title{
Correction Scheme for Scheduled Network Loss Rate Applied to UHV Transmission Trading
}

\author{
Yinya Zhang ${ }^{1}$, Shunming Bai ${ }^{1}$ and Yukun Bao ${ }^{2}$ \\ 1. Central China Branch of Power Exchange Center, State Grid Corporation of China, Wuhan 430077, China \\ 2. School of Management, Huazhong University of Science and Technology, Wuhan 430073, China
}

Received: October 31, 2014 / Accepted: December 31, 2014 / Published: March 31, 2015.

\begin{abstract}
The size and allocation method of transmission network loss have major impact on particular users involved with the electricity market trading process, therefore, the accuracy of scheduled network loss rates will be vital to the economic concerns from the stakeholders. In view of the shortcoming of existing loss rate scheme in 1,000 kV demonstration project, three scheduled loss rates applied to the UHV AC (ultra high voltage alternating current) transaction settlement are revised. By taking relationship between loss rate and power into consideration, the new segmented correction scheme greatly reduces the deviation between scheduled loss rates and actual loss rates, and is more accurate, flexible and expansible than the old one.
\end{abstract}

Key words: UHV, loss rate, transaction settlement, curve fitting, loss allocation.

\section{Introduction}

The energy resources and productivity show an extremely non-uniform distribution in China, and the energy-rich regions are far from the economic developed regions. So the UHV (ultra high voltage) transmission, which is suit for long-distance, high-capacity and low-loss power transmission, might be the main transmission technology in China $[1,2]$. Nowadays, the construction of strong smart grid based on UHV as the backbone has been established as the State Grid's development strategy. The first $1,000 \mathrm{kV}$ UHV AC (ultra high voltage alternating current) transmission demonstration project, which is from $\mathrm{CZ}$ (Changzhi) to JM (Jingmen) via NY (Nanyang), has the world's highest operating voltage level and is the most complicated power transmission technology currently. This project uses south-north mutual operation modes, that is to say, it transports thermal power from Shanxi Province to central China (north-to-south mode) during dry season and takes

Corresponding author: Yinya Zhang, senior engineer, research fields: power exchange and power market. E-mail: zhangyy@cc.sgcc.com.cn. hydropower from central China to the North China (south-to-north mode) in rainy season.

Loss rate is an important synthesis technical and economic index of power grid company, and it is also an important factor which determines the price and settlement amount in electricity market [3-5]. In the monthly planning and transaction settlement of the $1,000 \mathrm{kV}$ UHV power exchange, the factor of network loss rate must be taken into consideration. Scheduled network loss rate should be considered to calculate the power price or amount, so the accuracy of the UHV scheduled network loss rate has direct impact to the economic interests of the stakeholders involved.

\section{Status of the UHV Transaction Settlement and Management of Net-Work Loss}

Currently, the CZ-NY-JM UHV transaction settlement is organized by the State Gird Corporation and is divided into two parts. The first is between the State Gird and CCPG (Central China Power Grid). In this case, the $\mathrm{CZ}$ node gateway electric quantity measurement can be transformed to CCPG's purchasing amount by network loss conversion while 
the network loss is borne by the State Gird. Secondly, the business is between the CCPG and the provincial power grid company based on the planned value of $\mathrm{CZ}$ side which can also be transformed into JM and NY sides. In the whole process of transaction settlement, three loss rates are involved. Take the south-to-north power transmission as an example with details below:

(1) Comprehensive network loss rate, which is calculated by the total loss of the UHV AC transmission system (including line loss and transformer loss) divided by the delivery charge at the beginning, is mainly used for converting the gateway data of $\mathrm{CZ}$ side to the NY/JM side, then the CCPG's trading amount can be calculated. The comprehensive loss rate takes a fixed value $1.5 \%$ currently;

(2) Henan loss rate, which refers to the network loss caused by the power transmitting to Henan from north China, is mainly used for converting the scheduled value of $\mathrm{CZ}$ to $\mathrm{NY}$ side, so the Henan Province's trading amount can be calculated. This loss rate is replaced by the CZ-NY line loss rate at present, the value is $1.2 \%$;

(3) The other provinces loss rate, which refers to the loss rate caused by the power transmitting to the other provinces (Hubei, Hunan, Jiangxi, Chongqing and Sichuan) except Henan in central China, is used to calculate the other provinces' settlement amount. Except Hubei, the settlement amount of Hunan, Jiangxi, Chongqing and Sichuan is the monthly scheduled value after loss converting to NY/JM side, and the deviation between scheduled value and actual value shall be borne by Hubei Province. The network loss caused by the power transmitting to Henan and the loss caused by other provinces should be equal to the comprehensive entire network loss, so the value of other provinces loss rate is currently derived from the two former loss rates, as a fixed number $1.52 \%$.

Although the scheduled network loss rate scheme is simple and clear by using a single and fixed value, but the following disadvantages exist:

(1) There is a relatively big difference between this scheduled network loss rate and the actual one, and the accuracy is poor for the actual loss rate value which varies with the changes of power amount. Currently, the three network loss rates related to the UHV transaction settlement all use fixed number. The inaccuracy of scheduled loss rate will directly lead to the inaccuracy of the trading electricity, thus affecting the fairness of the transaction;

(2) Errors exist when Henan loss rate is replaced by CZ-NY line loss rate because these two have different meanings, and are calculated by different algorithms. Note that specific error size will be specifically analyzed in Section 3.2.

\section{The New Correction Method for Scheduled Network Loss Rate}

\subsection{Correction of the Comprehensive Network Loss Rate}

In view of the inaccuracy of existing single fixed loss rate, this paper uses the fitting curve to correct the comprehensive network loss rate piecewise by taking the change of transmission power into consideration. The comprehensive network loss rate and monthly average transmission power of the UHV AC project from January 2012 to August 2013 are shown in Table 1 according to the gateway measured data since the NY substation is putting into operation.

As shown in Table 1, the UHV AC project, whose comprehensive loss rate stays between $1.4 \%$ and $1.5 \%$,

Table 1 Comprehensive network loss rate and transmission power of the UHV AC project ( 10 MW).

\begin{tabular}{llllll}
\hline Month & $\begin{array}{l}\text { Power } \\
(10 \mathrm{MW})\end{array}$ & $\begin{array}{l}\text { Loss } \\
\text { rate }(\%)\end{array}$ & Month & $\begin{array}{l}\text { Power } \\
(10 \mathrm{MW})\end{array}$ & $\begin{array}{l}\text { Loss } \\
\text { rate }(\%)\end{array}$ \\
\hline 2012.1 & 182.63 & 1.50 & 2012.11 & 196.20 & 1.32 \\
2012.2 & 200.26 & 1.46 & 2012.12 & 208.13 & 1.42 \\
2012.3 & 236.39 & 1.61 & 2013.1 & 231.38 & 1.60 \\
2012.4 & 217.64 & 1.46 & 2013.2 & 196.56 & 1.44 \\
2012.5 & 168.23 & 1.32 & 2013.3 & 234.67 & 1.43 \\
2012.6 & 103.89 & 1.57 & 2013.4 & 201.41 & 1.34 \\
2012.7 & 153.15 & 1.39 & 2013.5 & 174.04 & 1.44 \\
2012.8 & 158.19 & 1.46 & 2013.6 & 92.33 & 1.76 \\
2012.9 & 169.51 & 1.41 & 2013.7 & 87.82 & 1.83 \\
2012.10 & 86.82 & 1.75 & 2013.8 & 65.34 & 2.17 \\
\hline
\end{tabular}


has a relatively small transmission power is in the past two years. However, the biggest loss rate reaches $2.17 \%$ while the smallest one is only $1.22 \%$, so there is a big difference between the fixed $1.5 \%$ and the actual one.

Theoretical results show that, the network loss of UHV system is mainly composed in resistive loss and corona loss. The resistive loss is proportional to the square of current, and the corona loss is proportional to the square of the voltage, regardless of the current size [6-8]. Since the voltage of the UHV line mainly remains at $1,000 \mathrm{kV}$, the relationship between the comprehensive loss rate and the UHV transmission power should be a quadratic function that is confirmed by the scatter plot (Fig. 1) mapped according to Table 1.

Using the least squares method, the function of the fitting curve can be got as follows:

$$
\eta_{1}(P)=3.098-0.019 P+5.303 \times 10^{-5} P^{2}
$$

where, $\eta_{1}$ is the UHV compressive network loss rate, $P$ is the monthly average transmission power. From Fig. 1, it can be seen that, this curve well reflects the UHV comprehensive loss rate trend along with the change of transmission power. Considering the practicability and operability, this curve can be divided into several segments $\left[P_{1}, P_{2}\right],\left[P_{2}, P_{3}\right] \cdots\left[P_{i}, P_{i+1}\right], \cdots$ according to the size of transmission power, and the corresponding comprehensive network loss rate of the power interval $\left[P_{i}, P_{i+1}\right]$ is set as $\eta_{1}\left(\frac{P_{i}+P_{i+1}}{2}\right)$. When planning and transaction settlement are conducted, the staff can read more accurate UHV comprehensive loss rate just according to the interval to which the transmission power belongs.

Nowadays, in view of the facts that the transmission power of the UHV AC line is relatively small, and there may be bigger and smaller power, this paper expands the power range to $200-3,000 \mathrm{MW}$ and divides the range into four segments. Thus, the correction scheme of the comprehensive loss rate is achieved and can be shown in Table 2.

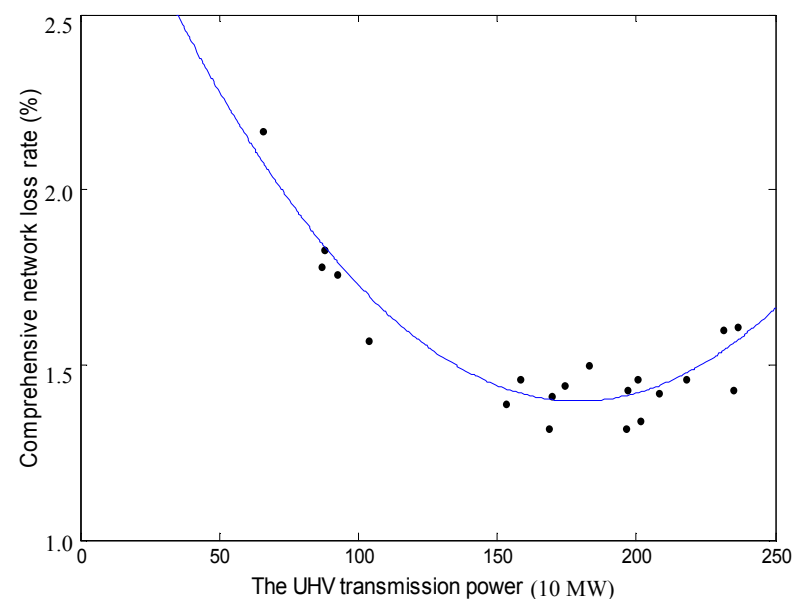

Fig. 1 The scatter plot of comprehensive network loss rate and transmission power.

Table 2 Correction scheme of the comprehensive network loss rate.

\begin{tabular}{lll}
\hline $\begin{array}{l}\text { Power interval } \\
(10 \mathrm{MW})\end{array}$ & Value & $\begin{array}{l}\text { Comprehensive } \\
\text { loss rate (\%) }\end{array}$ \\
\hline$[20,90]$ & $\eta(55)$ & 2.21 \\
{$[90,160]$} & $\eta(125)$ & 1.55 \\
{$[160,230]$} & $\eta(195)$ & 1.41 \\
{$[230,300]$} & $\eta(265)$ & 1.78 \\
\hline
\end{tabular}

\subsection{The Correction of the Henan Network Loss Rate}

\subsubsection{Error Analysis for Using the CZ-NY Line Loss} Rate

Set the planned electric quantity sent out from CZ's medium voltage side as $W_{1}$, the electricity sent to Henan province as $W_{2}$, and the others as $W_{3}$. The total loss of the UHV system is set as $\Delta W_{1}$, the loss caused by the electricity sent to Henan and the others are set as $\Delta W_{2}$ and $\Delta W_{3}$, respectively. The transformer loss of $\mathrm{CZ}, \mathrm{NY}$ and JM is $T_{1}, T_{2}, T_{3}$, respectively, and the CZ-NY line loss and NY-JM line loss is $L_{1}, L_{2}$ as shown in Fig. 2.

In addition, set the UHV comprehensive network loss rate as $\eta_{1}$, while the Henan loss rate and other provinces loss rate $\eta_{2}, \eta_{3}$. The CZ-NY line loss rate (CZ-NY loss includes CZ transformer loss, CZ-NY line loss and NY transformer loss) is set as $\eta_{C N}$. Thus, the calculation formula for UHV AC comprehensive network loss rate is written as:

$$
\eta_{1}=\frac{\Delta W_{1}}{W_{1}}=\frac{T_{1}+L_{1}+T_{2}+L_{2}+T_{3}}{W_{1}}
$$




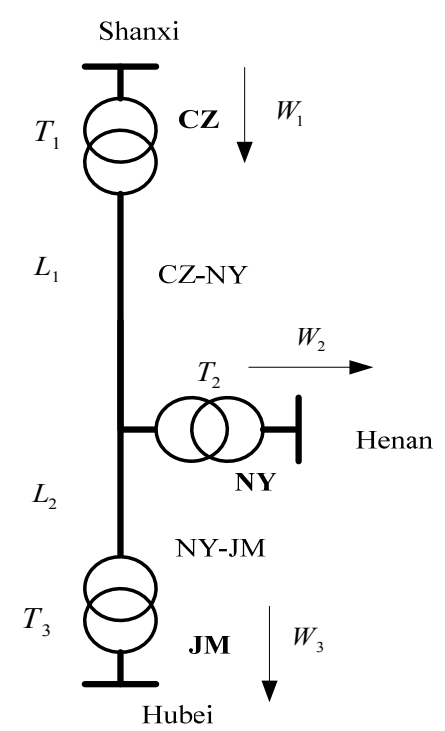

Fig. 2 The UHV AC line schematic diagram and the meanings of each variable.

It can be seen that, the UHV comprehensive network loss rate multiplied by the electricity sent out from the medium voltage side of $\mathrm{CZ}$ side is the accurate total loss $T_{1}+L_{1}+T_{2}+L_{2}+T_{3}$. How to select the value of Henan loss rate and the other provinces loss rate is actually a loss allocation problem. That is how the total loss reasonably should be apportioned to Henan and the other provinces in central China.

The Henan network loss rate calculation method is below if it is replaced by the CZ-NY line loss rate:

$$
\eta_{C N}=\frac{T_{1}+L_{1}+T_{2}}{W_{1}}
$$

According to Eq. (3), network loss caused by the electricity sent to Henan is:

$$
\Delta W_{2}^{\prime}=W_{2} \eta_{C N}=\frac{W_{2}}{W_{1}}\left(T_{1}+L_{1}\right)+\frac{W_{2}}{W_{1}} T_{2}
$$

The first item $W_{2} / W_{1} \cdot\left(T_{1}+L_{1}\right)$ on the right side of Eq. (4) means that, Henan allocates the CZ transformer loss and CZ-NY line loss according to proportion of the electricity sent out, which is actually a kind of power flow tracing method $[9,10]$. However, the NY transformer loss $T_{2}$ should be fully allocated to Henan, the second item $W_{2} / W_{1} \cdot T_{2}$ shows that Henan just shares a part of it, thus the error occurs.

As the superposition principle is not fit for power calculation in AC circuits, there is currently no method based on strict mathematical or physical theory in grid loss allocation [11]. The power flow tracing method can be used appropriately in UHV trading because it directly describes the actual trend of physical flow and is easily accepted by all trading members. According to the thought of allocate loss in proportion, the network loss caused by electricity sent to Henan and the others are as follows, respectively:

$$
\begin{gathered}
\Delta W_{2}=\frac{W_{2}}{W_{1}}\left(T_{1}+L_{1}\right)+T_{2} \\
\Delta W_{3}=\frac{W_{3}}{W_{1}}\left(T_{1}+L_{1}\right)+L_{2}+T_{3}
\end{gathered}
$$

In this way, fairness can be guaranteed and the loss equal principle $\left(\Delta W_{2}+\Delta W_{3}=\Delta W_{1}\right)$ can be satisfied. So the error caused by the replacing of CZ-NY line loss rate is:

$$
\Delta W-\Delta W_{2}^{\prime}=T_{2}-\frac{W_{2}}{W_{1}} T_{2}=W_{3} \frac{T_{2}}{W_{1}}
$$

Set $T_{2} / W_{1}=\lambda$, so the network loss rate caused by electricity sent to Henan should be:

$$
\Delta W_{2}=W_{2} \eta_{C N}+W_{3} \lambda
$$

where, $W_{3} \lambda$ is the term of error or correction of electricity loss, $\lambda$ is correction coefficient. That is to say, the reasonable value can be got if we add a correction term when using CZ-NY line loss rate to replace the Henan loss rate. Meanwhile, we can see from Eq. (8) that the branch line electricity loss is not only associated with the power passing through itself, but also the other branches, accurate value can not be got by using only one loss rate.

\subsubsection{Correction of Henan Network Loss Rate}

According to Eq. (8), the formula for calculating Henan network loss rate is:

$$
\eta_{2}=\frac{\Delta W_{2}}{W_{2}}=\eta_{C N}+\frac{W_{3} \lambda}{W_{2}}
$$

where, $\eta_{C N}$ is CZ-NY line loss rate and $W_{3} \lambda / W_{2}$ is the correction term.

At first, the condition that the CZ-NY line loss rate changing with the transmission is taken. From July to September at each year when UHV AC is operated in south-to-north mode (mainly the Sichuan hydroelectric 
power sent to the north), Henan does not involve the UHV trading, so the correction should be done just when the power flow is from north to south.

According the monthly CZ-NY line loss rate and UHV averaged transmission power, the same method is used as Section 3.1. By processing the comprehensive loss rate, the scatter diagram emerging the relationship between CZ-NY line network loss rate and transmission power is shown in Fig. 3 and the curve fitting function is Eq. (10):

$$
\eta_{C N}(P)=1.759-0.0065 P+1.235 \times 10^{-5} P^{2}
$$

Also, by extending the power range to 200-3,000 MW and dividing it to four intervals, each CZ-NY line loss rate of the power interval is set to its middle value. So Table 3 can be got.

The size of error term $W_{3} \lambda / W_{2}$ can be analyzed according to Eq. (9). Historical data from January 2012 to August 2013 indicate that, the error term $W_{3} \lambda / W_{2}$, which stays between $0.08 \%-0.11 \%$, is relatively stable. Here, we take the value as the average $0.1 \%$, that is

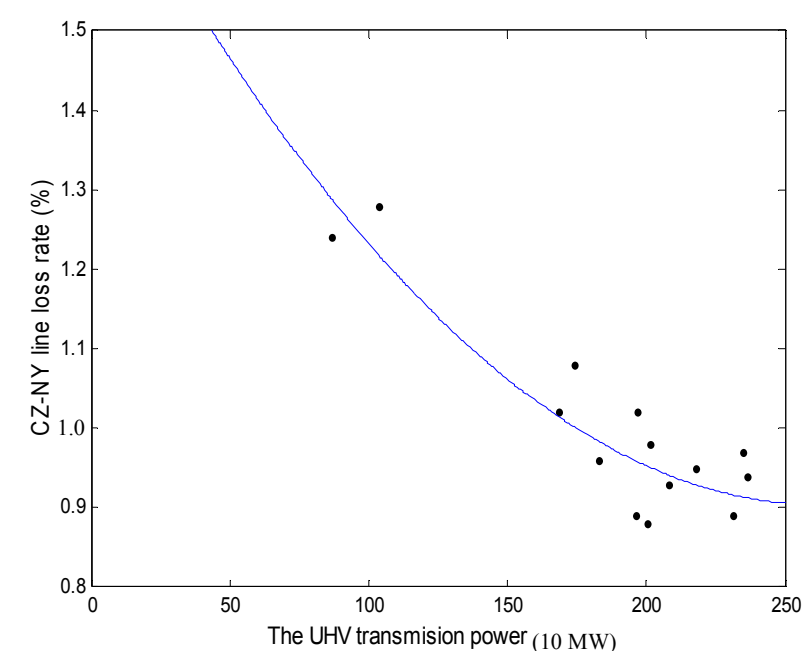

Fig. 3 The relationship between CZ-NY network loss rate and the UHV transmission power.

Table 3 Correction scheme of the CZ-NY line loss rate.

\begin{tabular}{lll}
\hline $\begin{array}{l}\text { Power interval } \\
(10 \mathrm{MW})\end{array}$ & Value & CZ-NY line loss rate (\%) \\
\hline$[20,90]$ & $\eta_{C N}(55)$ & 1.44 \\
{$[90,160]$} & $\eta_{C N}(125)$ & 1.14 \\
{$[160,230]$} & $\eta_{C N}(195)$ & 1.01 \\
{$[230,300]$} & $\eta_{C N}(265)$ & 0.90 \\
\hline
\end{tabular}

to say, when CZ-NY line loss rate is smaller than the Henan network loss rate, the error is about $0.1 \%$. Therefore, if we add this error as depicted in Table 3, the Henan network loss rate can be revised as shown in Table 4.

\subsection{The Correction of the Other Provinces Network} Loss Rate

The correction of provincial network loss rates other than Henan can refer to the processing method of Section 3.2. That is to replace the other provinces network loss rate with the CZ-JM line loss rate. But the method is complicated, and the CZ-JM line transmission power is difficult to describe due to the shunting action of NY node, so this paper uses the loss-equal principle to correct the network loss rates in other provinces.

Determining the value of Henan loss rate $\eta_{2}$ and the other provinces loss rate $\eta_{3}$ is actually a loss allocation problem as mentioned in Section 3.2, and rates $\eta_{2}, \eta_{3}$ with the UHV AC comprehensive network loss rate $\eta_{1}$ should obey the following loss-equal principle:

$$
W_{1} \eta_{1}=W_{2} \eta_{2}+W_{3} \eta_{3}
$$

When the UHV operates in south-to-north mode, Sichuan hydropower is totally sent to North China through UHV line while Henan is not involved with the trading, so $W_{2}=0, W_{1}=W_{3}$. That is to say, the other provinces network loss rate is equal to the UHV comprehensive network loss rate in Table 2.

When the UHV operates in north-to-south mode, $W_{2} \neq 0$, the calculation formula of the other provinces network loss rate is:

$$
\eta_{3}=\frac{W_{1} \eta_{1}-W_{2} \eta_{2}}{W_{3}}
$$

Table 4 Correction scheme of the Henan network loss rate.

\begin{tabular}{ll}
\hline Power interval & Henan network loss rate (\%) \\
\hline$[20,90]$ & 1.54 \\
{$[90,160]$} & 1.24 \\
{$[160,230]$} & 1.11 \\
{$[230,300]$} & 1.0 \\
\hline
\end{tabular}


Table 5 Correction scheme (north to south) of the other provinces network loss rate.

\begin{tabular}{ll}
\hline $\begin{array}{l}\text { Power interval } \\
(10 \mathrm{MW})\end{array}$ & $\begin{array}{l}\text { The other provinces loss rate } \\
\text { (north-south) }(\%)\end{array}$ \\
\hline$[20,90]$ & 2.28 \\
{$[90,160]$} & 1.58 \\
{$[160,230]$} & 1.45 \\
{$[230,300]$} & 1.86 \\
\hline
\end{tabular}

Table 6 Correction scheme of the three scheduled network loss rates in the UHV AC transmission trading.

\begin{tabular}{lllll}
\hline $\begin{array}{l}\text { Power } \\
\text { interval } \\
(10 \mathrm{MW})\end{array}$ & $\begin{array}{l}\eta_{1} \\
(\%)\end{array}$ & $\begin{array}{l}\eta_{2} \\
(\%)\end{array}$ & $\begin{array}{l}\eta_{3}(\%) \\
\text { (north-to-south) }\end{array}$ & $\begin{array}{l}\eta_{3}(\%) \\
\text { (south-to north) }\end{array}$ \\
\hline$[20,90]$ & 2.21 & 1.54 & 2.28 & 2.21 \\
{$[90,160]$} & 1.55 & 1.24 & 1.58 & 1.55 \\
{$[160,230]$} & 1.41 & 1.11 & 1.45 & 1.41 \\
{$[230,300]$} & 1.78 & 1.00 & 1.86 & 1.78 \\
\hline
\end{tabular}

According to the trading mode organized by the National Grid, central China provinces proportionally consume the thermal power from North China, the consumptive proportion between Henan and the other central provinces remains at about 1:9, that is:

$$
\begin{aligned}
& W_{2}: W_{3}=1: 9 \\
& W_{2}: W_{1}=1: 10
\end{aligned}
$$

Combining the Eqs. (12)-(14) along with the data in Tables 2 and 4, the corrected value of the other provinces network loss rate is gotten when the UHV operates in north-to-south mode.

By integrating the data shown in Tables 2-5, the correction scheme of three network loss rates in UHV AC transmission trading can be shown as in Table 6 . The staff in trading center can read more accurate UHV comprehensive loss rate just according to interval which the transmission power belongs to.

Where, $\eta_{1}$ is the comprehensive network loss rate, $\eta_{2}$ is Henan network loss rate and $\eta_{3}$ is the other provinces network loss rate.

\section{Conclusions}

In the transaction settlement of the UHV AC transmission, the amount and allocation method of transmission network loss have major impact on all users in the electricity market trading process, while the accuracy of scheduled network loss rates has the direct impact to the economic interests of all stakeholders. In view of the shortcoming of present loss rate scheme in $1,000 \mathrm{kV}$ demonstration project, three scheduled loss rates correction method are proposed and applied to the UHV AC transaction settlement in this paper. Advantages of this new scheme are as follows:

(1) The new method is fair and transparent with the objective historical data for analysis and allocating network loss by transmission electricity proportion, so it is easily accepted by the trade partners;

(2) It has a high accuracy and is more close to the actual one. By taking relationship of loss rate and power into consideration, the new segmented correction scheme greatly reduces the deviation between scheduled loss rates and actual ones;

(3) It has good scalability and extensibility. When UHV AC transmission power exceeds over the given range, the corresponding network loss rate can still be quickly determined according to the fitted curve. Besides, with the accumulation of the gateway statistical data, the fitting curve can be corrected and the segmented power range can be more refined to make the results more accurate according to actual needs.

\section{References}

[1] Jinglian, W., and Yongxi, X. 2005. "Development Prospect of UHV AC Power Transmission in China." Power System Technology 29 (3): 1-4.

[2] Yinbiao, S. 2005. "Research and Application of 1,000 kV UHV AC Transmission Technology." Power System Technology 29 (19): 1-6.

[3] Tao, W., and Jianmin, Z. 2003. "Calculation of Scheduled Loss Ratio and Its Assessment." Power System Technology 27 (7): 40-2.

[4] Fang, Z., Hai, B., and Yihan, Y. 2011. "A Biding Model for Day-Ahead Market Transaction Considering Modification of Network Loss." Power System Technology 35 (4): 164-9.

[5] Weiguo, Y., Youjing, X., and Xinfeng, Z. 2006. "Analysis on Technical Line Losses of Power Grids and Countermeasures to Reduce Line Losses." Power System Technology 30 (18): 54-63.

[6] Hanqing, L., Xiong, W., and Xuming, L. 2013. "Operation Losses and Economic Evaluation of UHVAC 
and HDVC Transmission Systems." High Voltage Engineering 39 (3): 630-5.

[7] Yunpeng, L., Shaohua, L., and Qifa, W. 2010. "Analysis of Corona Loss of UHV AC Single Circuit Test Line under Rain." In Proceedings of CSEE (Chinese Society of Electrical Engineering), 114-9.

[8] Zhengxiang, H. 2013. Power System Analysis (the Fifth Addition). Hanzhou: Zhejiang University Press.

[9] Kirschen, D., and Strbac, G. 1999. "Tracing Active and
Reactive Power between Generatiors and Loads Using Real and Imaginary Currents." IEEE Transactions on Power Systems 14 (4): 1312-9.

[10] Zihong, L., Haozhong, C., and Xiaodong, L. 2004. "Review of Loss Allocation Methods in Power Market." Relay 32 (7): 70-6.

[11] Naichao, C., and Zhizhong, G. 2003. "Pondering on the Loss Allocation Problem." In Proceedings of the CSEE, 43-7. 